Musées, Patrimoine et Culture scientifiques et techniques

$153 \mid 2014$

mai-juin 2014

\title{
Les muséums et le récolement : des bases communes pour une stratégie déclinable
}

Joël Clary et Nathalie Mémoire

\section{OpenEdition \\ Journals}

Édition électronique

URL : http://journals.openedition.org/ocim/1360

DOI : $10.4000 /$ ocim. 1360

ISSN : 2108-646X

Éditeur

OCIM

Édition imprimée

Date de publication : 25 juin 2014

ISSN : 0994-1908

Référence électronique

Joël Clary et Nathalie Mémoire, «Les muséums et le récolement : des bases communes pour une stratégie déclinable », La Lettre de l'OCIM [En ligne], 153 | 2014, mis en ligne le 25 juin 2016, consulté le 01 mai 2019. URL : http://journals.openedition.org/ocim/1360 ; DOI : 10.4000/ocim.1360 


\section{Les muséums et le récolement : des bases communes pour une stratégie déclinable}

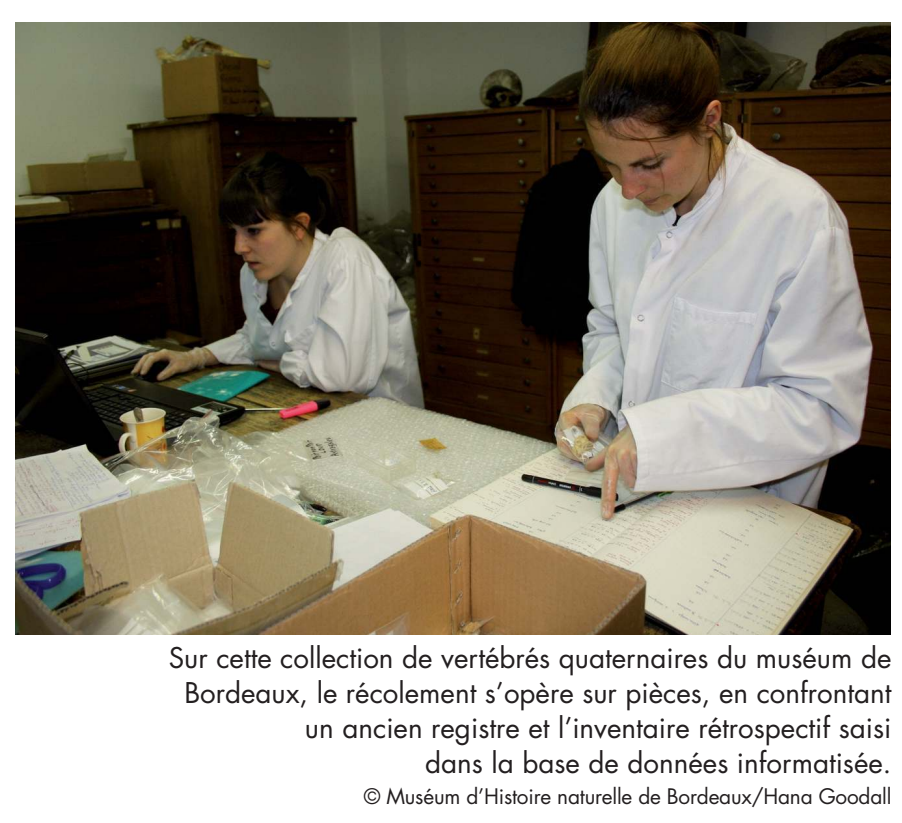

Joël Clary est conservateur du patrimoine au musée des Confluences à Lyon joel.clary@rhone.fr Nathalie Mémoire est conservateur en chef du patrimoine, directrice du muséum d'Histoire naturelle de Bordeaux n.memoire@mairie-bordeaux.fr

\section{Joël Clary et Nathalie Mémoire *}

À partir de la présentation de l'évolution des pratiques de gestion des collections des muséums et en particulier de leur inventaire, les auteurs font le constat des différentes stratégies et méthodologies employées, dressent un état des lieux des opérations du récolement décennal - montrant sa complexité mais aussi sa contribution à la connaissance des collections - et envisagent les perspectives $d^{\prime}$ avenir ainsi que les prolongements possibles de cet exercice.

Les muséums d'Histoire naturelle en France regroupent des musées dédiés aux sciences naturelles proprement dites (zoologie, géologie, paléontologie, botanique) ainsi que, depuis leur création, des collections de sciences humaines (préhistoire, archéologie et ethnographie). En y ajoutant les anciens «musées mixtes » qui comprenaient à la fois des collections d'Art et des collections d'Histoire naturelle, la plateforme OCIM-muséums créée en 2011 (voir le texte de Patrice Charon, p. 26) a identifié 68 établissements dont 65 ont fourni des données : 39 d'entre eux sont aujourd'hui membre de la Conférence permanente des Muséums de France créée le 26 janvier 2011 (voir l'encadré de Pierre Pénicaud, p. 15).

Héritiers des cabinets de curiosités, puis des cabinets d'Histoire naturelle pour les plus anciens, plus de la moitié des muséums ont été créés avant 1900 et près de $20 \%$ sont antérieurs à 1800 . Ils sont sous la tutelle du ministère de l'Éducation nationale, de l'Enseignement supérieur et de la Recherche (MENESR). En effet, lors de la création du ministère de la Culture en 
1959, les muséums sont restés au sein du ministère de l'Éducation nationale ; ils sont logiquement passés sous tutelle du MENESR (en 1982, ils sont sous tutelle de la direction des bibliothèques, des musées et de l'information scientifique et technique, DBMIST, au sein du ministère de l'Éducation nationale : voir le texte de Roland Bertrand et Francine Fontanel p. 23), celui-ci regroupant des attributions qui faisaient historiquement partie de l'Éducation nationale. Les muséums d'Histoire naturelle conservent la tutelle du MENESR mais depuis la loi de 2002, ils ont généralement obtenu l'appellation « Musée de France » et de ce fait se sont engagés à en respecter les obligations et donc le contrôle du ministère de la Culture, relayé en région par les DRAC (voir le texte de Myriam Boyer, p. 25). La conduite du récolement décennal fait partie intégrante de ces obligations.

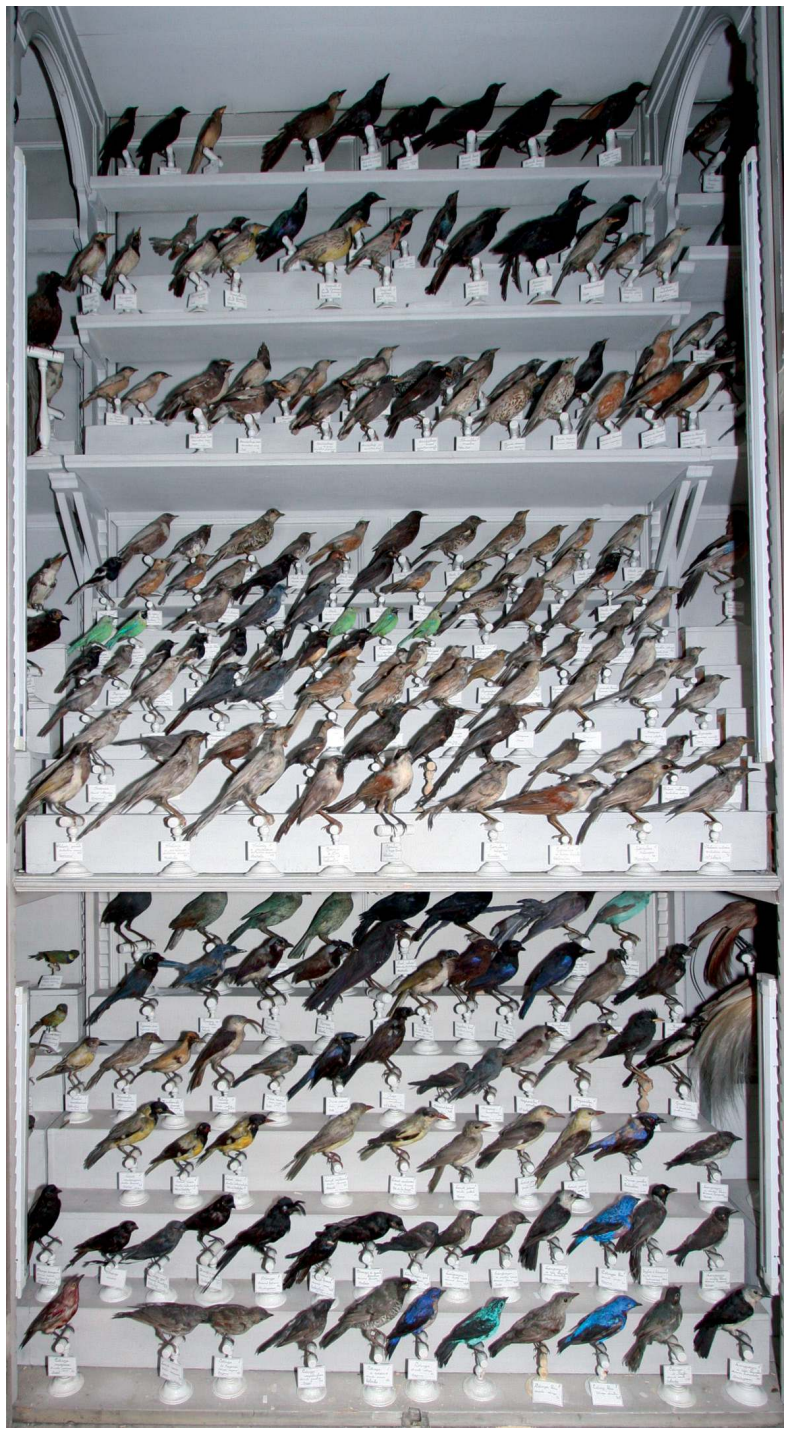

Une partie de la « collection générale » d'oiseaux exposée au muséum de Bordeaux (de 1862 à 2009).

(c) Muséum d'Histoire naturelle de Bordeaux/Frédéric Deval.

\section{Inventorier la nature, pas les collections}

Durant plus de deux siècles, les collections d'Histoire naturelle ont été conçues comme un échantillonnage de la nature (aujourd'hui la biodiversité). Jusqu'au début du XXe siècle, la réalisation de l'inventaire exhaustif des choses de la nature semblait encore un objectif raisonnable. Depuis, l'accroissement de la connaissance a permis de relativiser cette ambition, la collection du musée figurant aujourd'hui une représentation bien partielle de la biodiversité.

Le souci d'exhaustivité qui prévalait un temps, entraînait parfois la sélection d'un nombre limité d'échantillons d'une même espèce, érigés en collections de référence, notamment dans les présentations au public, l'objectif étant de posséder le plus grand nombre d'espèces possible, pour les groupes les plus connus comme les vertébrés mais aussi pour les mollusques ou les insectes. Parallèlement, l'accumulation d'un grand nombre d'échantillons, n'offrait souvent pas le temps de les trier, de les classer et de les inventorier et se faisait au détriment de la documentation propre aux objets, allant parfois jusqu'à la substitution des listes d'objets par des listes d'espèces.

La pratique alors fréquente des « collections générales » consistait à regrouper des spécimens provenant d'entrées au musée bien distinctes, dans une logique de rangement strictement taxinomique. Cette pratique facilitait la lecture scientifique de la collection mais elle a pu entraîner la perte de l'origine des pièces ou rendre leur recherche longue et difficile, notamment pour les insectes.

Jusqu'au milieu du XXe siècle, beaucoup de spécimens étaient considérés comme renouvelables, ce qui conduisait parfois à remplacer un spécimen par un autre, du moins pour les espèces répandues, faisant fi de la notion de bien unique. Cependant, certains biens remarquables étaient déjà élevés au rang d'objets patrimoniaux, en premier lieu les «types », spécimens de référence ayant servi à décrire et nommer les espèces, ou encore les spécimens d'espèces menacées ou disparues. Pour pallier le manque de matériel de référence, une autre pratique a perduré jusquau $\mathrm{XX}^{\mathrm{e}}$ siècle. Elle consistait en l'échange de matériel, généralement entre institutions et parfois aussi avec des particuliers ; les traces de ces mouvements n'ont malheureusement pas toujours été clairement consignées.

Le contexte n'est aujourd'hui plus le même. La connaissance de la biodiversité s'est considérablement améliorée et affinée en même temps que la prise de conscience de sa fragilité, ce qui a progressivement fait évoluer le regard sur les collections. Les objets de muséums, objets témoins de la diversité biologique, sont aussi les marqueurs de son état, récent ou plus ancien, 
et de sa dégradation. Ainsi, à la nature purement scientifique des objets s'est adjointe progressivement une dimension patrimoniale entraînant une évolution des pratiques de gestion des collections. Lorientation patrimoniale de la loi «Musée de France » a conforté cette tendance.

\section{De l'inventaire documentaire à l'inventaire règlementaire}

\section{L'état des lieux}

Les spécificités des collections de sciences naturelles, tant par leur nature, leur importance et leur complexité que par leurs modes de gestion, ont rendu difficile la mise en œuvre des obligations du récolement. Ceci est vrai pour les obligations primordiales du récolement comme la vérification de la présence des biens en un lieu ou leur conformité aux sources documentaires, mais aussi pour d'autres aspects du récolement comme le marquage, l'état des biens...

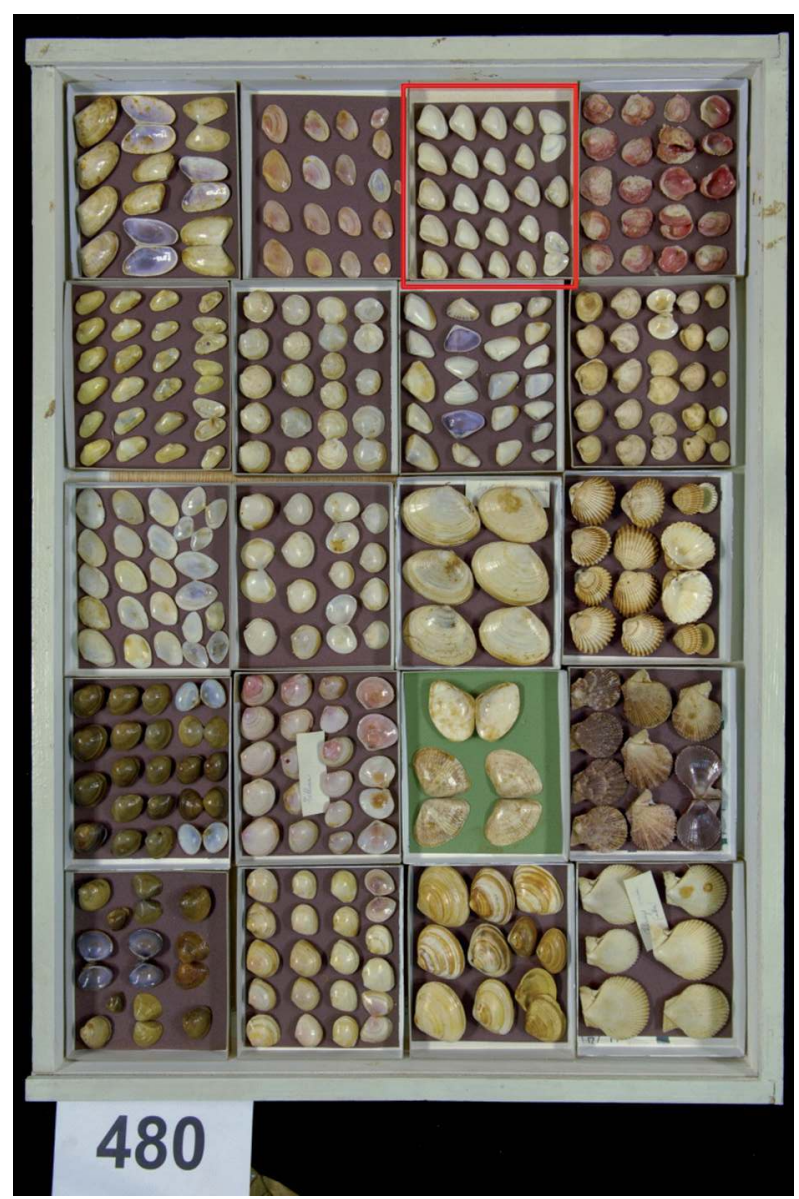

Comment compter ces coquilles de la collection Moreau du muséum de Bordeaux ? 1 plateau, 20 caissettes ou 298 spécimens? Le plateau ( $n^{\circ} 480$ ) est l'unité à considérer pour le récolement par lots complexes, 20 est le nombre de lots simples à considérer pour l'inventaire scientifique et la saisie dans la base de données informatisée, 298 est l'unité pertinente pour le comptage du nombre total des spécimens collections. ○ Muséum d'Histoire naturelle de Bordeaux/Frédéric Deval
Mais qu'entend-on par biens ? Se pose notamment la question de la pertinence du choix de l'unité utilisée pour le récolement et l'inventaire réglementaire. Selon les catégories de collection de sciences naturelles, un bien peut être apprécié de différentes manières. Pour une collection de coquilles ou d'insectes par exemple, doit-on prendre en compte le spécimen, le lot de spécimens (plusieurs individus de même espèce, même provenance...), l'ensemble d'un plateau ou d'une boîte ? Selon les cas, les durées de traitement ne seront bien sûr pas identiques! Ce choix a en outre une répercussion immédiate dans le chiffrage du nombre d'objets (voir le texte de François Dussoulier, p. 38). Pour s'en faire une idée : un muséum important affichera plusieurs centaines de milliers de biens si l'unité d'inventaire se limite à la boîte d'insectes ou à la liasse d'herbier mais ce chiffre passera à plusieurs millions dans le cas où l'inventaire s'opère au spécimen. La dimension minimale de l'échantillon (en minéralogie, pétrographie, micropaléontologie...) peut aussi influer sur le résultat du comptage (voir le texte de Muriel Lecouvez, p. 42). L'expérience montre que l'importance numérique des collections est généralement surévaluée au départ des estimations mais s'affine à la baisse au fur et à mesure de la progression du taux d'inventaire des collections (voir l'encadré d'Agnès Villain, p. 40).

Est-on en mesure de faire le lien entre le bien et son origine, autrement dit son entrée administrative en collection ? Récoler les objets de collection s'opère à partir de documents écrits, au premier rang desquels figure l'inventaire. L'immense majorité des muséums ne possèdent pas un inventaire unique et normalisé. En revanche, ils détiennent pour certaines de leurs collections des catalogues que l'on peut assimiler à des inventaires documentaires. Ce ne serait pas un handicap majeur s'ils étaient complets et cohérents entre eux, ce qui est rarement la règle. Ces inventaires se présentent sous diverses formes, même au sein d'une même institution. Parfois même, les muséums disposent de listes taxinomiques d'espèces et non de spécimens, parfois de listes de spécimens sans numérotation ou dont la numérotation n'est pas reportée par un marquage sur le spécimen.

En raison des usages courants dans les muséums, ces inventaires privilégient bien souvent les données scientifiques comme la date et le lieu de la collecte, le nom du collecteur, au détriment des données administratives comme le nom du donateur, la date et les modalités d'entrée des objets en collection (voir le texte de Brigitte Rollier p. 45). Parfois ces inventaires n'existent pas et ce sont les étiquettes associées aux spécimens qui portent toute l'information documentaire (voir le texte de Ludovic Besson, p. 43). 


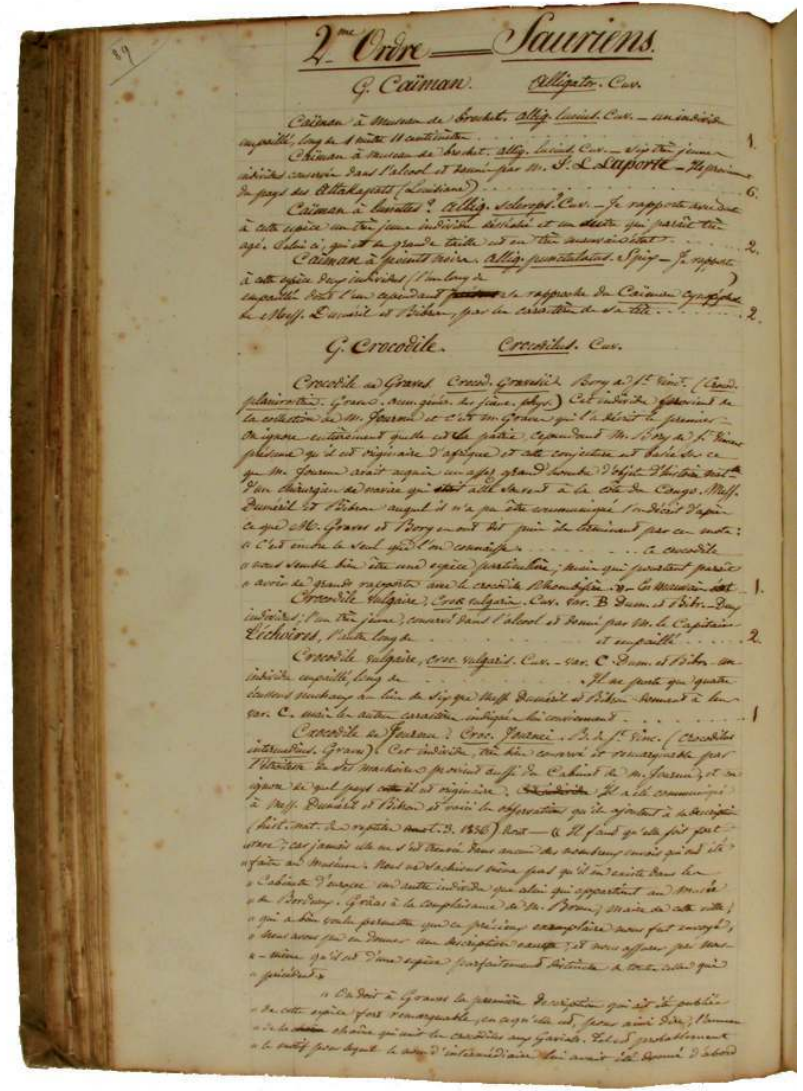

Au muséum de Bordeaux, le registre des collections du Cabinet d'Histoire naturelle (document au moins antérieur à 1853) est tenu selon la logique de la collection générale, selon un classement strictement taxinomique. II s'agit de listes sans numéros d'inventaire. Sur cette page, les deux spécimens décrits correspondent à des "types » : ils sont les seuls spécimens rapportables à ces espèces de crocodiles ; leur donateur (Bernard Journu Auber) est connu ainsi que la date du don (1804). Mais dans le cas d'espèces communes, ce document ne permet pas le rapprochement avec tel ou tel spécimen des collections.

๑) Muséum d'Histoire naturelle de Bordeaux/Bernard Fontanel

Depuis Carl von Linné (Systhema naturæ, 1758), la désignation des biens en Histoire naturelle, s'effectue sous la forme de noms scientifiques selon une nomenclature dite binominale (genre et espèce). Ceux-ci changent au cours du temps en fonction de l'évolution des connaissances et leur actualisation peut s’avérer délicate lorsque, dans les inventaires, le nom réactualisé est venu se substituer au nom d'origine sans que ce dernier ait été conservé. Pour la plupart des muséums, les contenus du ou des inventaires scientifiques, ainsi que les données portées sur les étiquettes, lorsque celles-ci sont restées associées au spécimen, ont servi de base à la constitution de la base de données informatisée des biens. Celle-ci est un outil complexe qui doit intégrer les spécificités d'exploitation liées à la nature et au mode d'exploitation des données d'Histoire naturelle. Un bon exemple en est la mise au point et l'évolution des thesaurus de classification scientifique.

Le travail d'inventaire documentaire a pu être réalisé selon les cas en interne lorsque les compétences scientifiques étaient disponibles, ou en faisant appel à des

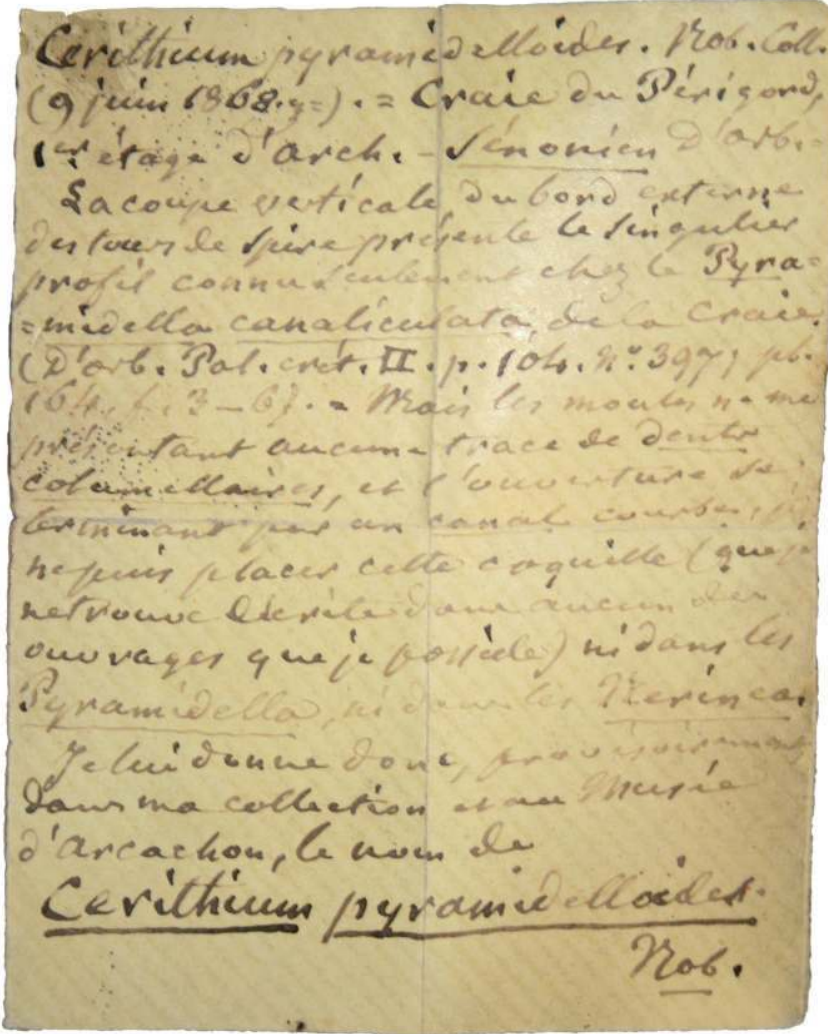

Cette étiquette, associée à un fossile de Cerithium pyramelloïdes porte toute l'information scientifique documentaire correspondant à ce spécimen. Lors de l'inventaire rétrospectif, ce type de document est scanné et inséré dans la notice informatisée. ○) Muséum d'Histoire naturelle de Bordeaux/Marie Jullion

spécialistes par discipline. Lorsque ceux-ci sont intervenus dans divers établissements, ils ont contribué à l'enrichissement des notices documentaires par croisement des sources issues des différents musées et ont favorisé ainsi l'harmonisation des pratiques (voir le texte de Marie-Françoise Faure et Laurent Charles, p. 33). Cette démarche d'inventaire documentaire a longtemps été soutenue par le ministère de tutelle, le ministère de l'Éducation nationale, de l'Enseignement supérieur et de la Recherche, dans le cadre de la politique d'informatisation des inventaires de collections, tandis que des préconisations de standardisation des données étaient fournies par l'OCIM (voir les textes de Roland Bertrand et Francine Fontanel, p. 23 et de Pierre Dalous, p. 47).

La bonne tenue des registres d'entrée facilite grandement la réalisation du récolement. Malheureusement, lorsqu'ils ont été conservés, ceux-ci sont souvent lacunaires et peu précis. La désignation des objets ou des ensembles d'objets figure généralement de manière succincte ou 


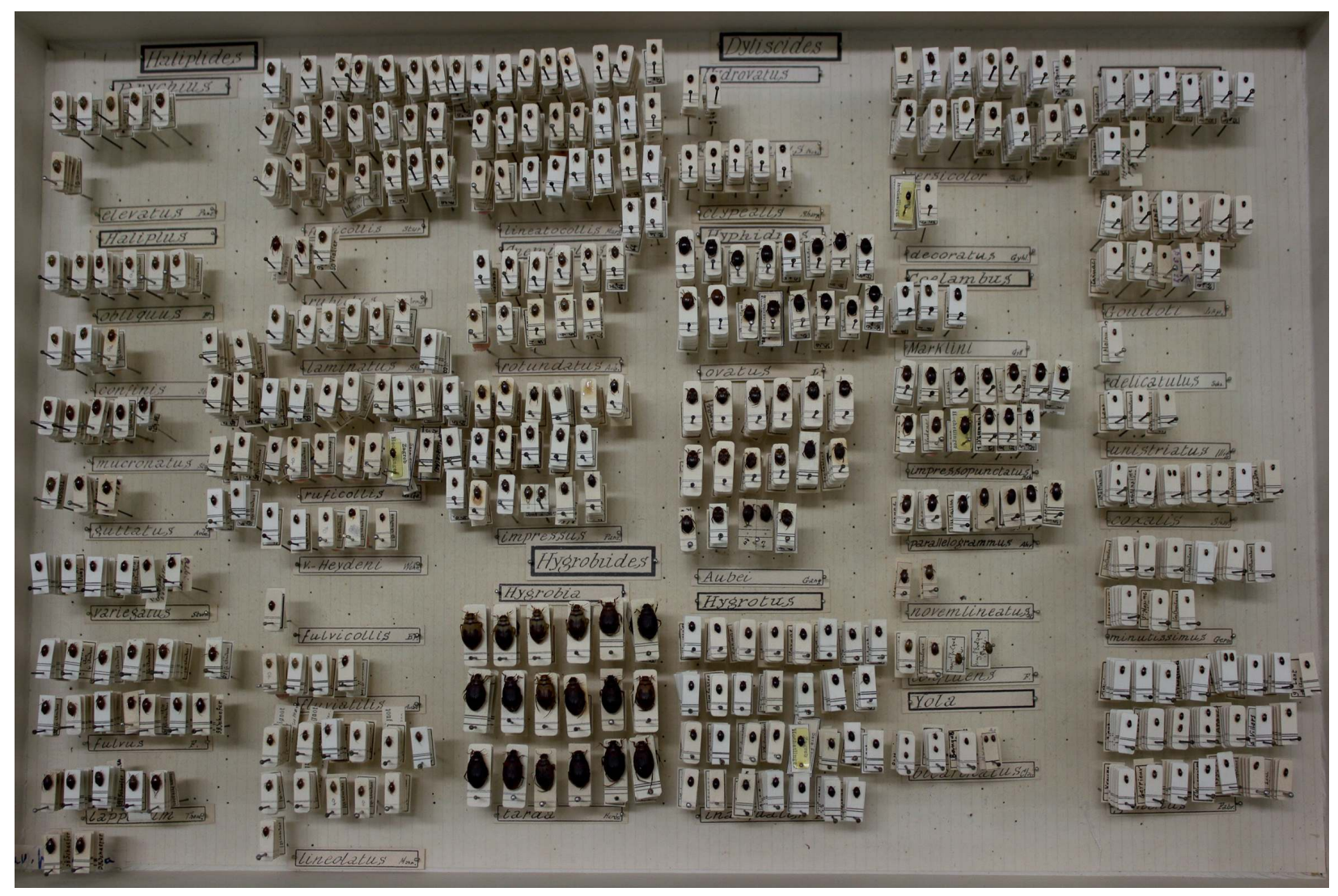

La stratégie de récolement : ce carton de coléoptères renferme 1249 spécimens épinglés en « brochettes » correspondant à 300 lots de collecte et représentant 42 taxons. Pour l'instant, il a été référencé et récolé avec un numéro d'inventaire unique (Collection G. Audras, musée des Confluences, inv. 460254). ๑) Département du Rhône/musée des Confluences/Joël Clary

peu précise (ex : don d'un lot d'oiseaux). Ces lacunes et ces imprécisions, notamment la confusion entre information de collecte et information d'entrée, rendent difficile l'exploitation de ces registres, le lien entre les entrées et les biens.

La localisation de l'objet fait partie des données réglementaires du récolement. Chaque objet devrait ainsi être localisé assez précisément. En termes de pratique, on sait que, dans beaucoup de muséums, l'habitude a longtemps été de favoriser le repérage des biens de manière visuelle, les spécimens étant rangés selon un ordre de classement scientifique bien établi, rendant accessoire la nécessité d'une cotation fine du mobilier de rangement. Néanmoins, les muséums qui ont dû réaliser des inventaires rétrospectifs face aux vitrines, en particulier dans la perspective de rénovations d'établissement et/ou d'aménagement de nouvelles réserves, ont été très tôt amenés à créer une cartographie et une typologie détaillées de leurs locaux et mobiliers afin d'enregistrer les localisations dans les notices des bases de données informatisées (voir le texte de Nathalie Mémoire, p. 59).

Si la pratique du marquage des pièces est bien ancrée dans les musées d'Art, elle l'est beaucoup moins dans les muséums, à cause de la nature même des objets, de leur petitesse, du grand nombre de pièces à traiter. Pour cette raison, les marquages s'opèrent bien souvent non sur l'objet mais sur les étiquettes fixées sur les socles, attachées aux spécimens ou simplement disposées auprès des spécimens. Ces modes de marquage ne s'avèrent pas toujours sûrs et sont très souvent sources de mélanges ou d'erreurs à répétition.

Un enjeu important du récolement est de dresser un état sanitaire des collections. Là encore, les muséums n'ont pas le recul historique des musées d'Art, notamment dans l'établissement des constats d'état. On le ressent, ne serait-ce qu'au niveau de la terminologie qui varie d'un établissement à l'autre. En revanche, et paradoxalement, les muséums ont souvent été précurseurs dans l'identification globale des dommages et la mise en place des mesures de conservation préventive. Dans ce domaine, ils ont alors souvent joué le rôle d'établissements ressources pour les autres musées (voir le texte de Sébastien Minchin, p. 61).

Enfin, le récolement ne s'applique qu'aux objets dont l'institution est propriétaire. Cette règle n'est pas propre aux muséums, mais, pour un certain nombre d'entre eux, des opérations de rapatriement de bribes de collections d'Histoire naturelle provenant d'autres institutions, comme des musées mixtes, des universités, des 


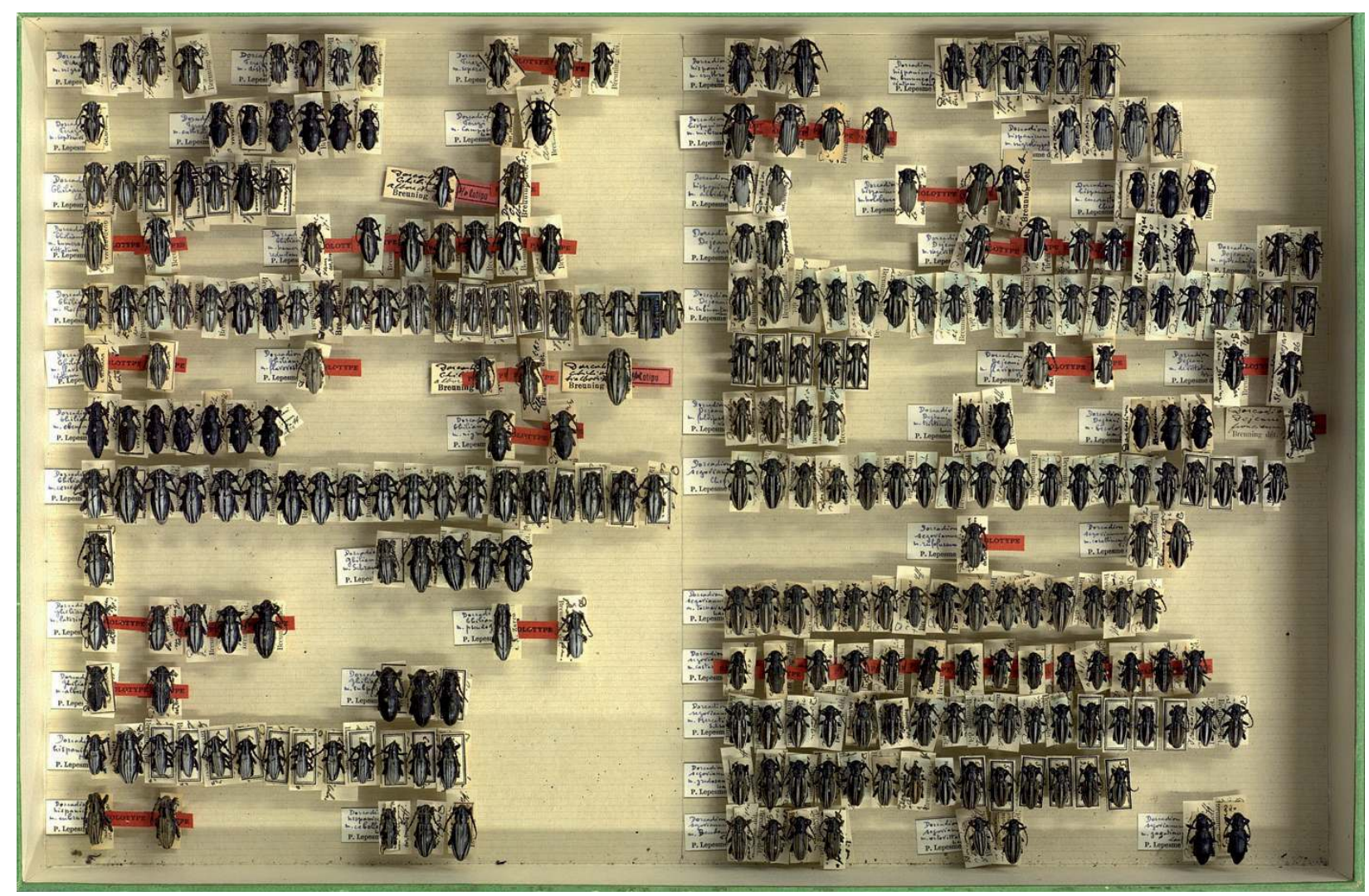

L'inventaire des biens remarquables : au sein des collections, les types sont conventionnellement repérés par des étiquettes rouges (Fonds entomologique P. Lepesme, musée des Confluences, inv. 468843). ๑) Département du Rhône/musée des Confluences

institutions religieuses ou le Muséum national d'Histoire naturelle, parfois traitées dans l'urgence, n'ont pas toujours été accompagnées d'une procédure formalisée de don ou de dépôt. Il conviendrait, en toute logique, de clarifier ces situations à l'occasion du récolement.

Des stratégies pour le récolement

Lapplication tardive des dispositifs réglementaires du récolement s'explique pour partie par les difficultés auxquelles ont été confrontés les musées en général et les muséums en particulier, à la différence d'autres établissements pour lesquels cette pratique du récolement est ancienne et classique comme dans les bibliothèques publiques ou les services d'archives (voir le texte d'Agnès Vatican, p. 29). Néanmoins, partout le travail a été engagé et le récolement est apparu comme l'occasion de procéder à un certain nombre d'opérations apportant une meilleure connaissance et accessibilité des collections. Il a été à la fois un sujet de questionnements et de réflexions, mais aussi de solutions et de réponses.

Les questionnements les plus fréquents concernent : - la manière de récoler les ensembles réunissant de grandes masses d'objets ;

- le mode d'exploitation de sources disparates, imprécises ou absentes ;
- le traitement des inventaires et des listes scientifiques existantes face à l'absence d'un inventaire unique et conforme, autrement dit l'adaptation des inventaires documentaires existants en un inventaire réglementaire ;

- la manière de traiter les objets et collections qui n'ont jamais été inventoriés ;

- la place à donner aux biens remarquables que sont les «types».

D'autres interrogations concernent les modalités de marquages complémentaires, la nomenclature de localisation, le traitement photographique des objets...

Face à ces questionnements, la diversité des réponses et des stratégies retenues est à l'image de la disparité observée entre les établissements, tant au niveau de leur taille, de leur mode de fonctionnement que du contexte local dans lequel ils évoluent. Les stratégies mises en œuvre par les uns et les autres dépendent bien sûr des niveaux d'inventaire et d'informatisation de leurs collections. Pour certains, le travail préparatoire était déjà largement engagé mais avec des règles et des nomenclatures différentes qu'il a été nécessaire de faire évoluer. Des musées ont complété les inventaires existants, parfois par une saisie simplifiée permettant de traiter un grand nombre d'objets. D’autres musées, dont 
Le 26 janvier 2011, l'OCIM organisait à Paris une rencontre professionnelle sur le thème Nowvelle Gouvernance de la Culture scientifique en France : nouveaux enjeux pour les Muséums de France. Des échanges de cette journée sont nés la Conférence Permanente des Muséums de France (CPMF) et son engagement à participer à l'élaboration de la plateforme des muséums créée par l'OCIM dans le cadre de sa mission d'Observatoire du patrimoine et de la culture scientifiques et techniques (OPCST) qui lui a été confiée par le ministère de l'Éducation nationale, de l'Enseignement supérieur et de la Recherche, en accord avec l'université de Bourgogne.

Ainsi, les représentants des 28 muséums présents se sont constitués en Conférence Permanente des Muséums de France avec pour objectif de porter la voix des muséums auprès des différentes instances et interlocuteurs du domaine. Elle est également un espace d'échange et d'élaboration de réflexion et de portage de projets collectifs.

Ses membres ont choisi d'adhérer à une charte d'objectifs et de règles de fonctionnement et regroupent aujourd'hui 39 muséums signataires (soit la presque totalité des établissements français) qui se rencontrent en réunion plénière deux fois par an.

Tous les membres, dans leurs actions, leurs représentations et leurs communications, agissent pour leur institution, bien sûr, mais aussi au nom de la communauté des muséums de France.

Les échanges se font au niveau des directeurs, mais tous les personnels participent grâce à un forum, animé par Michèle Lemaire, directrice du muséum de Bourges : la « museumliste ». Cette dernière, très active et regroupant plus de 160 professionnels, permet de transmettre les informations, de partager expériences et pratiques et de lancer des chantiers de réflexion. C'est aussi un outil de travail pour chercher des conférenciers, des spécimens, des informations scientifiques. .

Les nombreuses rencontres des représentants de la CPMF avec les différents acteurs nationaux ont permis de faire reconnaître l'importance des muséums dans le paysage.
La CPMF est maintenant l'interlocuteur incontournable et représentatif auprès :

- des 3 ministères directement impliqués (ministère de l'Éducation nationale, de l'Enseignement Supérieur et de la Recherche-MENESR, ministère de l'Écologie, du Développement durable et de l'Énergie-MEDDE, ministère de la Culture et de la Communication-MCC) ; - d'Universcience et du Muséum national d'Histoire naturelle-MNHN (qui est membre de la CPMF) ;

- des associations (Association des musées et des centres pour le développement de la culture scientifique, technique et industrielle-AMCSTI, Association générale des conservateurs des collections publiques de FranceAGCCPF, International Council of Museums-ICOM) ; - des élus (Maires de France, Assemblée Nationale, Sénat). Ces actions communes se concrétisent :

- simplification pour les muséums des démarches concernant les espèces protégées et sensibilisation du MEDDE sur le rôle qu'ont les muséums sur les questions de société ;

- travail reconnu sur le récolement décennal par le MCC ; - implications des muséums dans les sciences participatives (Vigie nature) MNHN ;

- nombreuses citations ou sollicitation de la CPMF dans divers rapports, notes, articles (Service des musées de France, AGCCPF, OCIM...) ;

- demandes d'intervention de la CPMF (Forum de la CSTI, journées d'étude, Institut national du patrimoine...).

Le travail collaboratif avec l'OCIM se poursuit dans l'organisation des rencontres professionnelles de la CPMF, dans le travail de l'OPCST et dans un projet important de création d'une plateforme pour l'itinérance des expositions, dans le cadre des investissements d'avenir. En trois ans, la CPMF a ainsi gagné une véritable lisibilité dans le paysage de la culture et du patrimoine scientifiques et grâce à elle, les muséums confortent leur place légitime et historique.

Pierre Pénicaud Conservateur en chef $d u$ patrimoine, Directeur adjoint du département des galeries, Muséum national d'Histoire naturelle les catalogues n'étaient pas des sources fiables, ont procédé à un travail d'inventaire rétrospectif face aux vitrines ou aux rayonnages, ce qui a nécessité la création initiale d'une nomenclature de localisation. Toutes ces opérations ont été pour certains l'occasion de repérer les biens remarquables comme les «types » taxinomiques. Enfin, des musées ont choisi de procéder en deux phases successives : une première permettant d'affiner la documentation, de compléter les inventaires et de lier le maximum de biens à leurs origines, suivie d'un pointage final des biens en collection.

Devant l'ampleur de la tâche d'inventaire, beaucoup de musées ont profité de l'opportunité de traitement par ensembles complexes cohérents de parties de collections numériquement importantes, par exemple des liasses d'herbier, des cartons d'insectes, des plateaux de coquilles, des tiroirs de fossiles ou des collections nominales entières (par auteur, par gisement). 
La journée de travail, organisée le 26 mars 2013 dans les locaux de la DRAC Île-de-France (en présence de Sylvie Müller, chef du Service des musées, Laurence Isnard et Pauline Lucet, conseillers musées), avec le soutien de l'OCIM (représenté par Louis-Jean Gachet, directeur et Patrice Charon, chargé d'études), nous a permis de poursuivre la réflexion commune autour du récolement. Elle avait pour objectif :

- de confronter les problématiques et méthodologies engagées pour la mise en œuvre du récolement ;

- de proposer si possible les lignes stratégiques communes ébauchées en décembre ;

- de proposer si possible des mises en forme harmonisées pour la présentation finale des résultats.

Vingt musées de la CPMF ont contribué à la réflexion soit par leur présence à la journée (Angers, Bordeaux, Bourges, Le Mans, Lille, Lyon-musée des Confluences, Montbéliard-muséum Cuvier, Nancy, MNHN, Nice, Rouen, Saint-Quentin-musée des Papillons), soit par les informations envoyées sur leur état d'avancement, stratégies ou questionnements (Bayonne, ClermontFerrand, Marseille, Montauban, Nantes, Orléans, Toulouse, Strasbourg).

Après un bref rappel des difficultés particulières liées aux collections d'Histoire naturelle, chacun a pu évoquer l'état d'avancement et la démarche engagée prioritairement. Les plans de récolement décennaux (PRD) ont majoritairement été déposés en 2010 ou 2011. Deux lignes stratégiques sont apparues : l'une vise à ne récoler que ce qui a pu être inventorié et l'autre consiste à donner une vision globale de la totalité des biens, et à poursuivre les inventaires détaillés dans les suites au récolement (après 2014).

Les échanges ont ensuite concerné différents points pratiques : la rédaction des procès verbaux, la question des unités de comptages (déjà longuement débattue lors du travail avec l'Observatoire du patrimoine et de la culture scientifiques et techniques de l'OCIM), le référentiel à considérer pour les « non vus », l’impression des inventaires informatisés... Afin de tenter une harmonisation dans la présentation des résultats, et malgré les points d'interrogation qui subsistent, un lexique commun a été rédigé et validé.

\section{Le lexique}

Objet $=$ bien $=$ unique ou lot associé à un numéro d'inventaire et potentiellement à une notice.

Référence $=$ terme généraliste, équivalent à un numéro d'inventaire ; se rapporte à une fiche $=$ notice = un item .
Spécimen = individu au sens zoologique. Attention à l'ostéologie où un même spécimen comprend plusieurs parties (exemple un crâne et deux tibias d'un même individu peuvent correspondre à un lot ou à trois biens car trois numéros d'inventaire) ; c'est le cas exceptionnel où le nombre de spécimens peut être inférieur au nombre de biens...

Échantillon = «spécimen » en géologie.

Bien unique $=$ un spécimen unique avec un numéro unique (ex : un oiseau naturalisé, un mammifère).

Lot simple $=$ lot cohérent (plusieurs spécimens de la même espèce, de la même occurrence - date, provenance, mode collecte - , parties d'un même spécimen...) associé à un numéro d'inventaire et une notice (ex : un lot de coquilles d'œufs). À l'intérieur de ce lot simple (ex. 2002.2156.0), un spécimen remarquable (type ou autre) peut être isolé avec le numéro d’inventaire décliné (2002.2156.1).

Lot complexe $=$ lot souvent défini par un contenant (ex : boîte d'insectes, tiroir de coquilles, liasse d'herbier...), qui devra dès que possible être détaillé en plusieurs lots simples ou biens uniques pour la rédaction de notices. Peut être associé à un numéro d'inventaire provisoire ou définitif, décliné selon le niveau d'homogénéité du lot complexe. Il est recommandé de quantifier le plus précisément ce lot complexe en nombre de lots simples à venir et d'estimer le nombre total de spécimens représentés.

Ensemble complexe = ensemble important composé de plusieurs lots complexes (meuble thématique, collection nominale, gisement paléontologique). On s'efforcera de définir et de quantifier les lots complexes qu’il représente.

Objet inventorié = objet numéroté, marqué, référencé dans un support quelconque : registre manuscrit, tableur Excel, base de données informatisée (Actimuseo, SN-Base...). Attention des objets peuvent être inventoriés dans les bases sans appartenir pour autant aux collections « Musée de France « (les objets renouvelables ou « d'usage » dits de matériel pédagogique par exemple). Dans ce cas, ils doivent être clairement identifiables (par exemple par la nomenclature du numéro) car ils n'entrent pas dans le récolement.

Objet déterminé $=$ objet associé à une étiquette fournissant une ou des données documentaires (identification, provenance, origine...) qui permettra assez aisément un inventaire rétrospectif, mais non numéroté selon une liste utilisable comme référence. 
Objet non déterminé $=$ pas de documentation associée (étiquette) : nécessite une étude pour identification. Quelle pertinence pour ces objets? N'a pas pu être abordé en détail bien que la question du tri ait été posée. Il faudra y revenir. A priori, non concerné par le récolement dans l'immédiat.

Objet récolé $=$ comprend les objets vus et non vus.

Vus $=$ dont on a effectivement constaté la présence et l'état à une date donnée et dont on peut fournir la localisation.

Non vus = ce que recouvre ces « non vus » reste peu clair : quelle est la date et le document de référence en l'absence d'inventaire (dans le cas des inventaires rétrospectifs) ? Comptage des étiquettes orphelines ?
Des boîtes vides ? La mention des véritables manques identifiés (vols, destructions, pertes) est de toutes façons à noter dans les procès verbaux.

Nombre compté/nombre estimé = essayer de bien distinguer les objets comptés des nombres estimés. Lorsquau final on additionne comptés + estimés, refaire les arrondis.

Nathalie Mémoire pour la Conférence Permanente des Muséums de France

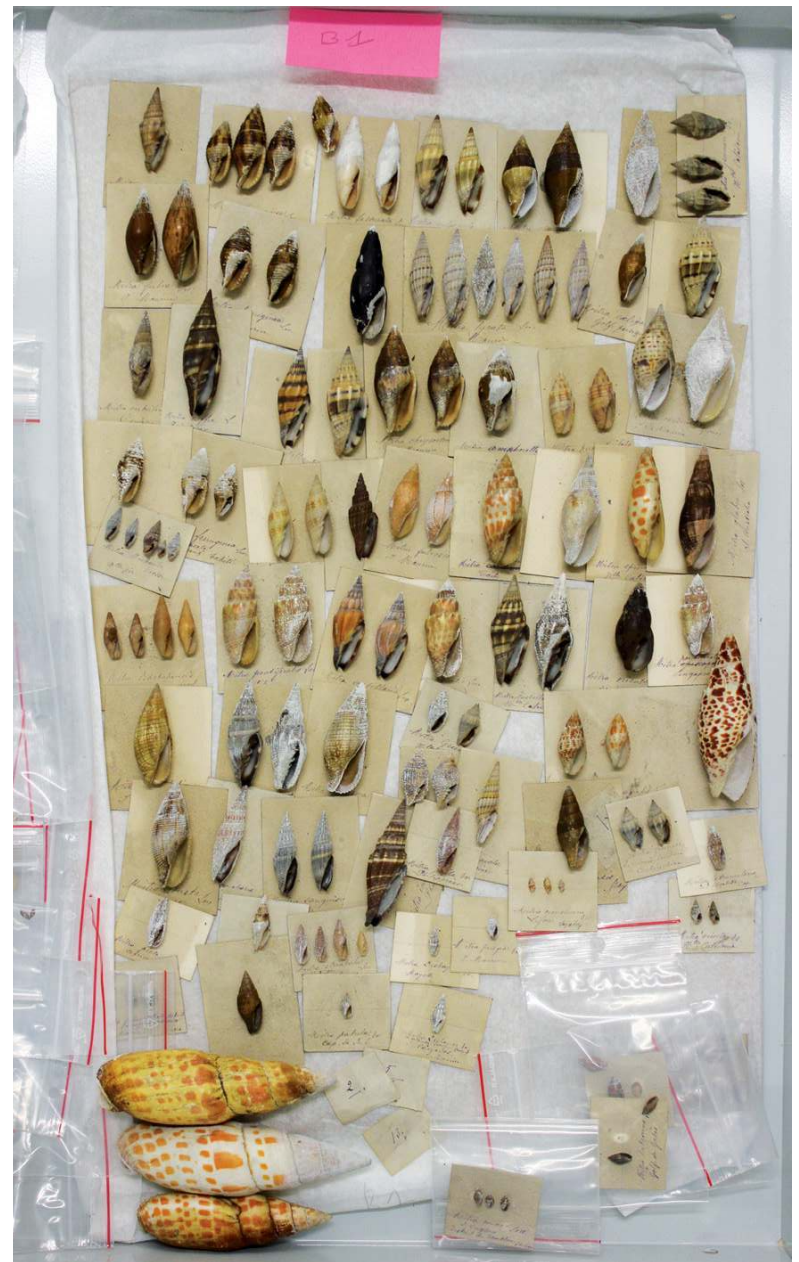

Le matériel d'étude : ces coquilles font partie d'un ensemble de matériel d'étude avant son traitement de patrimonialisation.

Pour éviter d'accentuer le mélange des échantillons et la perte d'information, les spécimens « vagabonds » ou " orphelins » ont été mis dans des sachets zip (Collection G. Roüast, musée des Confluences). ๑) Département du Rhône/musée des Confluences
Parfois, des chantiers des collections ont été mis en place. Ceux-ci ont permis une chaine de traitement des objets pour l'inventaire, le marquage, l'état sanitaire, la couverture photographique, le dépoussiérage ou le reconditionnement des objets. Ceux-ci ont souvent été motivés par d'autres objectifs, liés à la vie de l'établissement mais menés avec des calendriers partiellement décalés : projets de nouvelles réserves, mutualisées ou non (voir le texte de Pierre-Antoine Gérard et Sandra Delaunay, p. 51), projets de réaménagement de locaux (voir le texte de Pascale Joannot, p. 57) ou de rénovation complète d'établissement (voir le texte de Nathalie Mémoire, p. 59). Lors de ces chantiers, des questions collatérales ont surgi, notamment celles liées à la conservation préventive et aux questions d'hygiène et sécurité (voir le texte de Sébastien Minchin, p. 61). Enfin, d'autres questionnements ont vu le jour avec la publication de la note-circulaire relative aux matériels d'étude qui, après de nombreuses années de gestation, a fini par être publiée en date du 19 juillet 2012. À quels types de collections l'appliquer et selon quelles modalités concrètes ? Certains ont choisi de prendre en compte ce matériel d'étude, même s'il n'est pas censé figurer dans les procès-verbaux de récolement. Cette démarche a comme avantage de poser clairement la question du tri du matériel, de sa destruction et de la démarche de patrimonialisation (voir les textes de Gilles Cheylan, p. 68 et de Joël Clary, p. 73). Les réflexions ont pu être partagées avec d'autres musées gérant également de gros volumes de collections issus de modes de collecte comparables et apportant d'autres réponses (voir le texte d'Antoine Chancerel, p. 75).

\section{L'engagement de la CPMF dans la stratégie du récolement}

La réflexion collective engagée par les muséums, d’abord de façon informelle, mais stimulée par l'OCIM, puis 
organisée à la suite de la création de la Conférence Permanente des Muséums de France (CPMF), a été menée dans le cadre de réunions puis de journées de travail dédiées, d'échanges et enquêtes rendus possibles par le réseau de diffusion des muséums.

Ainsi, le 2 décembre 2010, l'OCIM sollicitait un petit groupe de 9 responsables de muséums pour débattre autour de son projet d'observatoire - plateforme collaborative et inventaire général. Décision était prise de s'engager dans un travail de mise au point d'une liste de rubriques pertinentes permettant de qualifier et quantifier les domaines d'activités et les moyens des muséums, en vue de fournir une image régulièrement actualisée de ceux-ci. Le projet et une première ébauche de cette liste étaient présentés, lors de la journée professionnelle organisée le 26 janvier 2011 qui conduisait à la fondation de la CPMF (voir l'encadré de Pierre Pénicaud, p. 15).

Les premiers résultats de la plateforme furent présentés à la CPMF le 27 janvier 2012 puis un nouveau groupe de travail, composé d'une dizaine de responsables de collections, fut réuni le 23 mars 2012 afin de retravailler la grille de rubriques, en particulier celles concernant le chiffrage détaillé des collections. À cette occasion, les échanges portèrent largement sur les modes de comptage en fonction de la nature des collections et l'avancée des inventaires.

Dans la suite logique de ces premières réflexions, la CPMF se proposa dès le début de l'été de répondre à l'appel à communication du Service des musées de France pour la Journée du récolement organisée à la BnF, à Paris, le 17 décembre 2012. Nous appuyant sur ces premières données du dispositif collaboratif (plateforme OCIM-muséums : données de 40 musées comparables sur les 51 répondants (voir le texte de Patrice Charon, p. 26), complétées par quelques échanges informels, nous avons choisi de présenter la problématique des muséums, dans toute sa complexité mais avec des pistes stratégiques permettant de proposer des réponses à l'obligation imposée par la loi « Musée de France ».

À nouveau avec le soutien de l'OCIM, nous avons poursuivi la réflexion commune lors d'une nouvelle journée de travail, le 26 mars 2013. Elle nous a permis de confronter les problématiques et méthodologies engagées, mettre en évidence les lignes stratégiques adoptées par les uns et les autres, enfin, élaborer un vocabulaire commun (voir l'encadré de Nathalie Mémoire, p. 16).

Toujours dans le cadre de cette démarche collective de la CPMF, nous avons pu répondre - grâce à l'efficacité du réseau de diffusion informel des muséums animé par Michèle Lemaire - à la demande d'intervention du Service des musées de France concernant le matériel d'étude (Journée récolement dans les musées nationaux

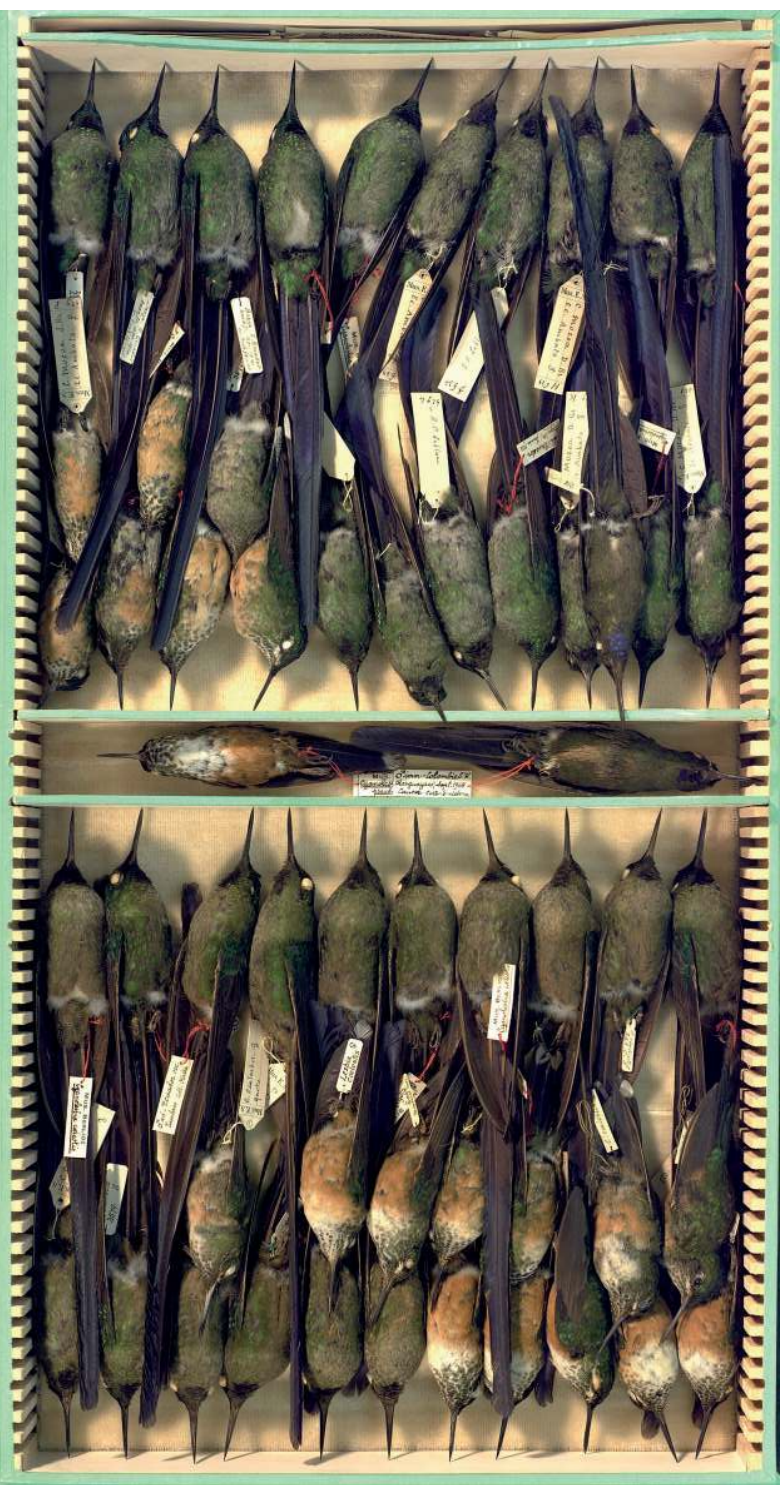

Les suites à donner : le récolement d'un ensemble complexe de colibris en peau s'est opéré au niveau du carton (186 au total). En revanche, l'inventaire et le marquage des 10000 spécimens sont inscrits dans les suites à donner au récolement (Collection E. Simon et J. Berlioz, musée des Confluences, inv. 419141). (c) Département du Rhône/musée des Confluences

du 18 octobre 2013 ; voir le texte de Nathalie Mémoire, p. 65), présentation renouvelée pour la session de formation permanente « inventaire et récolement » de l'INP (10-12 février 2014). Enfin, la CPMF a proposé une présentation de la valorisation du récolement dans les muséums pour la Journée du 12 décembre 2013. Cette dernière présentation n'a pu être retenue pour une question de calendrier mais fait ici l'objet d'un développement, abordant la diffusion dans les bases de données spécialisées (voir le texte de Marie-Dominique Wandhammer et Nicolas Morel, p. 79), les publications dans les revues (voir le texte de Thierry Malvesy, p. 86) ou les expositions grand public suite à des chantiers de collections 
(voir le texte de Sophie Rajaofera et Gilles Pavy, p. 90). Le 27 novembre 2013, à Dijon, nous avons présenté aux $2^{\text {es }}$ Rencontres de l'OPCST une brève synthèse de l'utilité et utilisation des données de la plateforme OCIM-muséums par des responsables de collections ou d'établissement.

Ainsi, les rencontres et échanges menés depuis plus de trois ans autour des problématiques liées aux collections ont permis de réunir, à chaque fois et à tour de rôle, entre la moitié et un quart des muséums, montrant ainsi l'investissement de tous dans cette volonté de partage. Ces échanges ont permis de recenser des difficultés, de confronter les problèmes et d'aborder aussi les stratégies développées par les uns et les autres, d'en proposer des ébauches de formalisation, enfin d'envisager des pistes de réflexions pour les suites à donner.

\section{Le taux d'avancement du récolement décennal}

Il est difficile de dire où en sont finalement les muséums à la veille de l'échéance du premier récolement décennal. Les indicateurs attendus par le ministère de la Culture via les DRAC ne permettent généralement pas de donner une image représentative de l'importance numérique des collections des muséums ni de l'ampleur du travail engagé. Les raisons en sont multiples.

Les muséums qui apparaissent ont pu répondre de différentes manières en fonction de la méthodologie de récolement qu'ils ont employée. En effet, ceux qui ont fourni des chiffres de biens récolés ou à récoler ont pu transmettre des chiffres correspondant tour à tour à des lots ou à des spécimens (voir « L'état des lieux », p. 13). Certains ont fait le choix de ne récoler que les biens qui étaient déjà inventoriés, ils disposaient donc d'un chiffre « cible » et ont pu fournir un taux d'avancement généralement conséquent (40 à $60 \%$ mais qui donne en fin de compte une image extrêmement sous-estimée du volume global de leur collection et ne rend pas compte de l'énormité du travail d'inventaire restant à réaliser. Les plus nombreux ont fourni comme " chiffre cible " l'estimation initiale du total des spécimens des collections et le taux d'avancement paraît généralement très bas (1 à $10 \%$ ). D'autres enfin n'apparaissent pas dans les statistiques car ils n'ont pas fourni le nombre de biens à récoler (chiffre « cible »). Parmi ces derniers, certains avaient choisi de traiter les grandes séries par ensembles complexes, chaque ensemble faisant l'objet d'une description et d'une simple évaluation du nombre d'items le composant. Pour ceux-là, le chiffre « cible » des biens (ceux déjà inventoriés et ceux qui resteront à inventorier dans les ensembles complexes), ne sera connu qu'à la fin de l'opération de récolement en cours. Ces musées n'ont donc pas été en mesure de fournir un taux d'avancement pertinent. Ils peuvent apparaître comme n'ayant pas commencé le travail alors qu'ils fourniront les résultats de l'opération globalement à l'échéance 2014.

Tout ceci montre combien la disparité dans le choix des unités de mesure et celui des stratégies de récolement rend difficile et délicate l'obtention et l'exploitation des chiffres d'avancement du récolement décennal pour les muséums.

\section{Les perspectives}

L'intention de ce premier récolement décennal est d'obtenir un état des lieux des collections qui doit permettre à chaque musée d'avoir une vision globale de ses biens et servir à l'établissement d'un inventaire réglementaire initial, dont il conviendrait en pratique de déposer une copie aux archives municipales ou départementales. Cet état des lieux doit rendre clairement compte de la situation des collections à la clôture du récolement, en précisant bien la méthodologie utilisée, afin de faciliter l'exploitation des résultats et la conduite des opérations ultérieures, des suites à donner. Selon la méthodologie adoptée et l'état d'avancement de leur récolement, ces tâches seront différentes d'un muséum à l'autre : inventaire des collections non inventoriées, documentation et inventaire fin des ensembles complexes, repérage des biens remarquables, opérations de marquage complémentaire...

En termes de bilan global, on peut souligner que, devant l'ampleur de la tâche, c'est l'aspect contraignant du récolement qui a bien sûr été perçu d'emblée et il convient de ne pas le minimiser au regard de la complexité des collections des muséums. On peut néanmoins considérer que le récolement a été pour beaucoup une opportunité à saisir pour se donner les moyens de faire progresser la connaissance des collections, des institutions, d'en souligner la richesse et l'importance. Un grand nombre de muséums l'ont perçu comme cela et, face aux difficultés mais aussi aux solutions apportées, la nécessité d'un partage d'expériences a émergé, même si cela s'est concrétisé trop tardivement. De ce point de vue, on peut dire que le récolement a catalysé les réflexions liées à sa mise en œuvre et a montré la capacité de réponse et d'adaptation des muséums.

Il convient maintenant de prolonger cette action, de continuer à travailler sur le partage d'expériences et l'harmonisation des pratiques, sans gommer les spécificités liées à la diversité des approches et des contenus. Cela concerne le récolement, mais également d'autres domaines qui sont au cour des préoccupations des chargés de collections : l'acquisition, la restauration, la documentation, l'archivage... Parmi les pistes évoquées, on peut mentionner : 
- la création d'une base de données « étiquettes » ébauchée durant l'été 2012 et qui resterait à construire ; - la standardisation, autant que possible, des procédures de renseignement des rubriques de la plateforme OCIMmuséums afin d'en rendre les données plus finement comparables ;

- la poursuite de l'organisation de journées de travail thématiques destinées à formaliser les pratiques communes les plus efficaces, comme les constats d'état, l'évaluation des valeurs d'assurance, les méthodes d'analyse des sources, la mise en place de l'inventaire réglementaire...

À un autre niveau et d'une manière plus générale, le récolement fait ressortir la nécessité de nourrir la réflexion sur la manière de concilier les missions scientifiques fondamentales des muséums et les contraintes liées aux nouvelles exigences patrimoniales. Ainsi, les collections des muséums ont vocation à être étudiées dans le cadre de programmes scientifiques de recherche ciblés et argumentés. Parfois, les modes d'intervention sur les objets dans le cadre de ces études peuvent apparaître en conflit avec le statut patrimonial des collections, lors des prélèvements à des fins d'analyse ADN, lors de la dissection des genitalia d'insectes afin de confirmer une identification. Ce qui est vrai pour un spécimen isolé, l'est également pour un échantillon dont l'étude au sein d'un ensemble peut nuire à sa qualité patrimoniale, de nature historique ou esthétique. Si l'évolution des outils d'investigation (scanner, IRM, tomographie...) permet aujourd'hui de préserver l'intégrité des objets, ce n'est le cas dans tous les domaines de la recherche. Dans ce contexte, comment concilier la préservation du patrimoine et soutenir le rôle scientifique joué par les collections sans le pénaliser par des procédures longues et fastidieuses ? La diversité des approches et des modalités de mise à disposition scientifique des collections, encourage à mener une réflexion sur ce sujet. 